\title{
SURVEY ON TECHNIQUES TO DETECT MICRO-CRACKS IN STEEL AFTER BENDING
}

\author{
B.De Smedt ${ }^{1}$, M. Abdel Wahab ${ }^{1}$, R. Hojjati ${ }^{1}$, K. Hertschap ${ }^{2}$ \\ ${ }^{1}$ Labo Soete, Ghent University, Belgium \\ ${ }^{2}$ ArcelorMittal Global R\&D, Gent, Belgium
}

\begin{abstract}
In this paper, different methods to detect micro-cracks were compared. Their capability of detecting fatigue induced micro-cracks in metal was evaluated, as well as the possibility to apply them to detect micro-cracks induced by bending in steel plates. All methods found in literature use one of four different physical phenomena. Out of these methods, the use of magnetic induced Eddy Currents and the use of Digital Image Correlation proved to be most interesting to detect micro-cracks in steel plates after bending. Also an FEA analysis was performed to detect the critical zones in bended specimens
\end{abstract}

Keywords: bending, steel, micro cracks, damage detection

\section{INTRODUCTION}

An important property of steel is the minimal bending radius. For construction, short bends are desired, but when metal is bend too short, micro-cracks appear. These micro-cracks form a big hazard in structural elements of machinery, as they are the cause of many fatigue failures. To determine the minimal bending radius for new materials, extensive research is needed to ensure strength, and thus safety, of bended plates. Most of this research is carried out with microscopes, without clear acceptance criteria.

Out of experience, the occurring micro-cracks originate in large numbers, with often small dimensions. In $90 \%$ of the cases, the cracks occur at the outside bend, the other $10 \%$, cracks are observed at the inner bend. These are the most strained regions during the bending. This is confirmed with an FEA, described in this paper. The zone of occurrence is a narrow strip beneath the bend knife.

In the light of fatigue damage, a lot of research is already carried out to detect micro cracks in steel plates. In this paper, a summery is made of existing detection techniques to determine their capabilities of detecting micro-cracks after bending. Four different sensor types are discussed, each using a different physical phenomenon. A fifth phenomenon, temperature, is also proposed, although not well documented in the literature.

\section{SURFACE ACOUSTIC WAVES (SAW)}

A first technique uses ultrasound waves to detect micro-cracks. The sound waves are focused on the surface with a water balloon, acting as a lens. The acoustic waves travel on the surface in the direction of the surveyed area (See Figure 1). Any discontinuity on the surface will reflect some of the surface acoustic waves (SAWs). These reflections are captured by a receiver. Out of the time difference, the distance to the discontinuity can be calculated. According to Wagle and Kato [1], this technique can detect cracks underneath other components, such as washers in nut-bolt connections. The technique is suited for larger areas.

Although the localisation of the crack is accurate, the smallest length of the detected crack mentioned was $290 \mu \mathrm{m}$. As the cracks after bending have smaller dimensions, the technique is not ideal to detect them. Also the large quantity of cracks will probably scatter the image. 


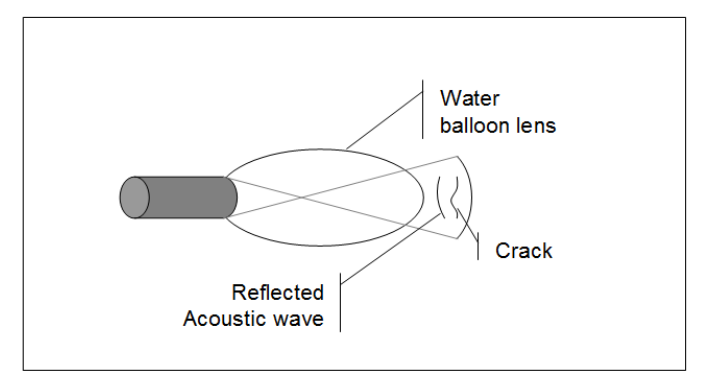

Figure 1. Surface Acoustic Waves are focused toward the surveyed area

\section{EDDY CURRENT SENSOR (EC)}

A second technique uses eddy currents in the material to detect micro-cracks. It uses a high frequency magnetic field to induce the eddy currents. The resulting magnetic induction is measured with a receiver spool. When a crack appears, it alters the electric properties of the surface. The eddy currents induced by the magnetic field change accordingly. This change is measured in the receiver spool (see Figure 2). With the combination of magnitude and the phase-shift, cracks can be detected.

According to Griesbach [2] and Goldfine [3], a crack of $50 \mu \mathrm{m}$ could be detected with permanently mounted sensors. With the large deformation of the specimen on the outside radius of the bend, special precautions have to be taken to prevent damage to the sensors. According to Goldfine [4] absolute values can be used, thus allowing measurements to be taken after bending.

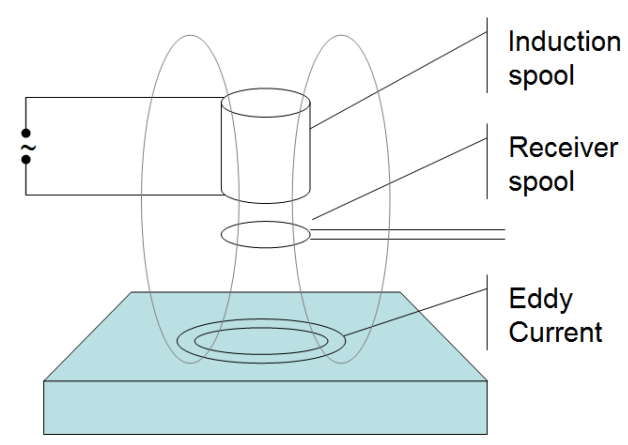

Figure 2. Electromagnetic field is altered because of Eddy Currents.

\section{MICROWAVES}

A third technique uses evanescent microwaves. The waves are radiated with a resonance probe (See Figure 3). The reflection of those waves on the surface is measured. When the sensor is positioned above a crack, the reflection is not strong. According to Kerouedan [5], the technique successfully detected 200 $\mu \mathrm{m}$ EDM notches, but the sensor proves to be very delicate. As the gap between sensor and surface has to be $50 \mu \mathrm{m}$, it demands a precision, 3-axis displacement device.

The effects of the unfinished surface of the bending samples makes it almost impossible to keep the $50 \mu \mathrm{m}$ gap

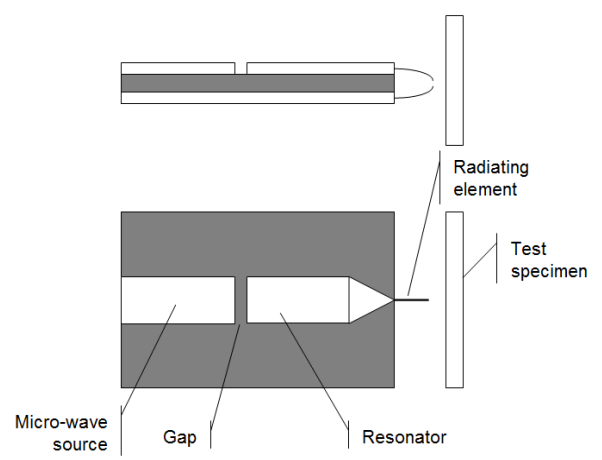

Figure 3. Micro-strip resonator design 
A last technique uses digital image correlation. Images are taken before and after loading the specimen. Using digital image correlation, the displacement vectors can be retrieved. The displacement can be predicted using FEA-methods, for an undamaged as well for a damaged specimen, as illustrated in figure 4. Using an algorithm, the displacement fields are compared with these patterns. Correlations with the latter indicate the presence of a micro-crack. The detectable cracks depend on the resolution of the camera as well on the fineness of the speckle pattern. Rupil [6] describes an experiment, which does not use a speckle pattern, but the surface is polished to ensure a clear image. It managed to detect cracks of 50 pixels or $160 \mu \mathrm{m}$ using only a 3MP camera to reduce computation time. As the computation time is not essential with the detection of micro-cracks in bended steel, the camera resolution can be picked higher to improve the detectable crack size.

The detected length might improve with a better camera to reduce the pixel size. In the case of bending, the nature of the samples might be a problem. As they must reflect the material under test, they can not be machined or polished. This means the mill scale is still present in some samples.

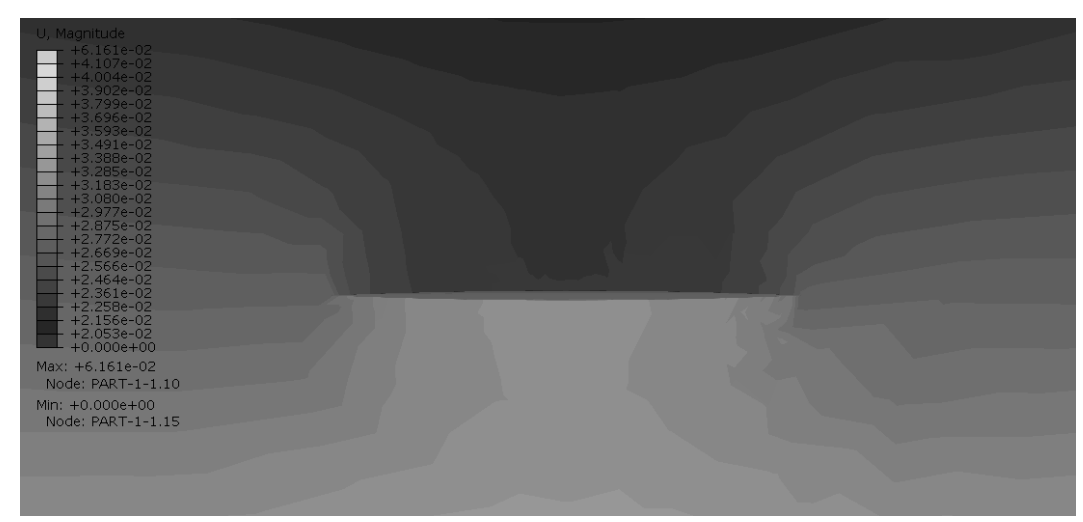

Figure 4. Simulated displacement field around a crack

\section{THERMAL IMAGING}

The use of thermal imaging to detect micro-cracks in metal is not described well in literature. Although basic simulations suggest a temperature difference occurs at a crack when applying a temperature difference over the specimen. Combining the technique with DIC might give good results, although mill scale and other impurities may obscure the image.

\section{FEA OF V-DIE BENDING}

To make a first estimation of the crack zone, a FEA is carried out. To solve the FEA, the program ABAQUS is used. A 2D-model of the sample is created as well as the bend knife and the V-die. The sample is a 6 $\mathrm{mm}$ thick plate of S235 steel. Besides the elasticity of steel, the plasticity behaviour of the material is entered in ABAQUS, as bending is a plastic operation. The knife has a radius of $8 \mathrm{~mm}$ and is modelled as an analytical rigid as the deformation of the knife is considered to be negligible. The V-die has a width of 50 $\mathrm{mm}$. The assembly is shown in Figure 5.

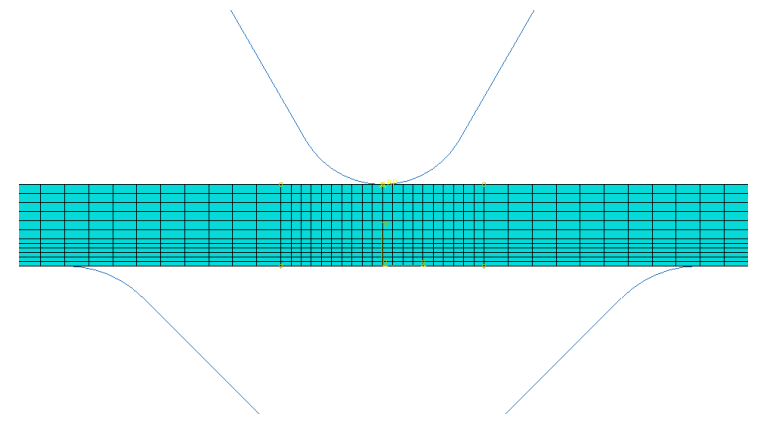

Figure 5. 2D model of the plate with analytically rigid knife and die

In this simulation, a force of $\mathrm{kN}$ is exercised on the knife, causing the plate to deform in to the V-die. This is shown in Figure 6. In this figure, we notice that the maximal stresses occur underneath the knife. Both on the top and bottom side of the plate plasticity occurs. As the topside is compressed, the likelihood of cracks is low. The bottom of the plate is stretched. This is the location were cracks are likely to occur. 


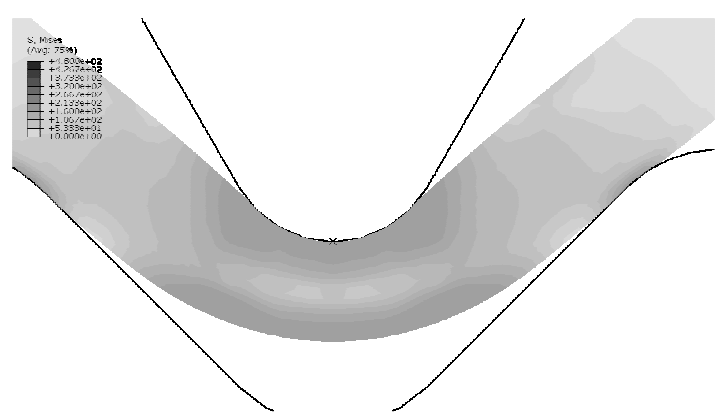

Figure 6: Stress in plate while bending

\section{CONCLUSION}

In the specific case of bending, EM looks most promising. The sensors are tested to detect small cracks. As they are surface mounted, special care has to be taken to prevent damage to the sensor. Using absolute values might prevent the need to mount the sensors while bending.

The DIC technique shows good results on machined surfaces. The raw surface of the samples used for test bending might prevent detection of the cracks.

Although $\mathrm{TI}$ is a potential technique for detecting cracks in metal, no literature has been found on the specific subject.

Both SAW and MW were found to be inadequate to detect micro-cracks on the samples.

From FEA results, we confirmed the critical zone where cracks will occur. The zone directly below the knife is most critical. The first cracks will appear in this zone.

\section{REFERENCES}

[1] Wagle, S. and Kato, H. (2010) Size Estimation of fatigue crack Appearing at bolt joints of aluminium Alloy Plates, Society for experimental Mechanics, (51), p.869-878

[2] Griesbach, T. et al. (2011) Design and fabrication of a modular Eddy Current Micro-Sensor. PIERS Proceedings, p.752-755.

[3] Goldfine, N. et al. (2002) MWM-Arry Eddy Current Sensors for detection of cracks in Regions with fretting damage. ASNT Materials Evaluation, 60 (7).

[4] Goldfine, N. et al. (2002) Surface Mounted and Scanning Periodic Field Eddy-Current Sensors for Structural Health Monitoring. IEEE Aerospace Conference proceedings, 6 p.3141-3152.

[5] Kerouedan, J. et al. (2006) Detection of surface Defects in Metallic Materials Using Evanescent Microwaves. ECNDT.

[6] Rupil, J. et al. (2011) Fatigue microcrack detection with digital image correlation. Gif sur Yvette cedex. 